\title{
Improved Dynamic Neighborhood Adaptive Particle Swarm Optimized Particle Filter for Integrated Navigation System
}

\author{
Zhimin Chen*, Yuming Bo, Yongliang Zhang, Yujian Li and Xiaodong Ling \\ ${ }^{1}$ China Satellite Maritime Tracking and Controlling Department, Jiangyin, \\ 214431, China \\ chenzhimin@188.com
}

\begin{abstract}
Particle filter based on particle swarm optimization (PSO-PF) is not precise enough and trapping in local optimum easily, it is not able to meet the requirement of modern navigation system. To solve the problems, a new particle filter based on dynamic clone particle swarm optimization (DPSO-PF) is presented in this paper. This improved filter enables the particles to fit the condition better and then reach the goal of global optimization through orthogonal initialization, clonal selection and local searching of self-learning, accordingly a best balance is achieved between optimal exploring and convergence rate. Finally univariate nonstationary growth model and integrated navigation model are used for simulation experiment and the results indicate that this new filter improves the precision of GPS/INS integrated navigation system.
\end{abstract}

Keywords: dynamic, particle filter, integrated navigation, clonal, orthogonal initialization

\section{Introduction}

With GPS position information and velocity information used for periodic calibration by INS, the performance of integrated navigation system can be superior to that of any sub-system used independently ${ }^{[1]}$. The system utilizes the highly accurate GPS information as the external measurement input. During the movement, modify INS and control its error as the time goes by ${ }^{[2]}$. However, in short term, the highly accurate INS positioning result can solve the signal unlocking and cycle slip problem in GPS dynamic environment. Besides, INS can assist GPS receiver to strengthen its anti-jamming capability, improve the capability of capturing and following the satellite signal. Therefore, GPS/INS combination system not only has small volume, light weight, and low power consumption, but also has the advantages of the high accuracy, low cost and strong anti-jamming capability, which is regarded as an ideal combination method in the current navigation domain.

In the indirect state estimation on the integrated navigation system, integrated navigation parameter error equation ${ }^{[3]}$ constitutes the main part of navigation system's state equation. On the basis of the some measurement output, it utilizes Kalman filtering to estimate different error status of the system, and uses the estimated value of the error state to correct the system, and then reach the target of the system combination.

Particularly, however, the civil and military field has made an increasingly higher demand $^{[4]}$ on the precision of integrated navigation system in recent years, given that the system noise and measurement noise may be the non-Gaussian noises, PF can be applied to non-linear system in the environment of non-Gaussian noise effectively since conventional $\mathrm{KF}$ is prone to divergence, but the existence of $\mathrm{PF}^{[5]}$ will result in sample 
degeneration and impoverishment and then exercise a severe influence on its state estimation performance.

The particle filter based on particle swarm optimization is one of the representatives of intelligent optimized PF. Through introduction of the latest system measurement to sample distribution, along with the utilization of particle filter for sampling optimization and constantly update of particles speed, the sample distribution is inclined to move to the region with higher posterior probability ${ }^{[6]}$. Particle swarm optimized particle filter improves the particle degradation of particle filter and is easier for actualization. But PSO-PF is a process of iterative optimization which will prolong the calculation time because of the high iterative frequency. Moreover, PSO-PF may be easily trapped into local optimization, influencing the accuracy and stability of practical engineering application $^{[7]}$.

The improved PSO-PF proposed herein carries out orthogonal strategy and dynamic adaptive clone particle swarm optimized algorithm for optimization, according enhancing the quality and diversity of particles. In this way, the filter can maintain not only high search ability, but also the diversity of particle swarm to heighten the locating accuracy of integrated navigation system.

\section{Particle Filter}

Particle filter is an approximate calculation of Bayes estimation based on sampling principle. It uses Monte Carlo method and Bayesian theory together, PF follows the principle thought that to find a group of sample for approximation of posterior probability density, replace the infinitesimal calculus in light of posterior probability density function by particle mean value, and thus acquire the minimal estimate of variance ${ }^{[8]}$.

Assuming the nonlinear dynamic process is defined as follows:

$$
\begin{array}{r}
x_{k}=f\left(x_{k-1}, v_{k-1}\right) \\
y_{k}=h\left(x_{k}, n_{k}\right)
\end{array}
$$

If the initial probability density of the system state is known as $p\left(x_{0} \mid y_{0}\right)=p\left(x_{0}\right)$, then the state predictive equation is defined as equation (3):

$$
p\left(x_{k} \mid y_{1: k-1}\right)=\int p\left(x_{k} \mid x_{k-1}\right) p\left(x_{k-1} \mid y_{1: k-1}\right) d x_{k-1}
$$

and the state renewal equation is:

$$
p\left(x_{k} \mid y_{1: k}\right)=\frac{p\left(y_{k} \mid x_{k}\right) p\left(x_{k} \mid y_{1: k-1}\right)}{p\left(y_{k} \mid y_{1: k-1}\right)}
$$

where

$$
p\left(y_{k} \mid y_{1: k-1}\right)=\int p\left(y_{k} \mid x_{k}\right) p\left(x_{k} \mid y_{1: k-1}\right) d x_{k}
$$

Supposing there is a known and importance function $q\left(x_{0: k} \mid y_{1: k}\right)$, which is rewrote as equation (6)

$$
q\left(x_{0: k} \mid y_{1: k}\right)=q\left(x_{0}\right) \prod_{j=1}^{k} q\left(x_{j} \mid x_{0: j-1}, y_{1: j}\right)
$$

Then the weight formula is

$$
w_{k}=\frac{p\left(y_{1: k} \mid x_{0: k}\right) p\left(x_{0: k}\right)}{q\left(x_{k} \mid x_{0: k-1}, y_{1: k}\right) q\left(x_{0: k-1}, y_{1: k}\right)}=w_{k-1} \frac{p\left(y_{k} \mid x_{k}\right) p\left(x_{k} \mid x_{k-1}\right)}{q\left(x_{k} \mid x_{0: k-1}, y_{1: k}\right)}
$$

To sample $N$ sample points $\left\{x_{k-1}^{i}\right\}_{i=1}^{N}$ from $p\left(x_{k-1} \mid y_{1: k-1}\right)$, then the probability density is as equation (8): 


$$
p\left(x_{k-1} \mid y_{1: k-1}\right)=\sum_{i=1}^{N} w_{k-1}^{i} \delta\left(x_{k-1}-x_{k-1}^{i}\right)
$$

And the weight of particles is:

$$
w_{k}^{i}=w_{k-1}^{i} \frac{p\left(y_{k} \mid x_{k}^{i}\right) p\left(x_{k}^{i} \mid x_{k-1}^{i}\right)}{q\left(x_{k}^{i} \mid x_{k-1}^{i}, y_{k}\right)}
$$

\section{PSO-PF Algorithm}

The importance sampling process of particle filter is not optimal, whereas the incorporation of PSO will optimize the sampling process of particle filter, it allow the weight of particle sets are more inclined to high likelihood area[9], accordingly solving the problem of particle impoverishment, and conducing to reduction of particle numbers required by particle filter. PSO algorithm is fused with PF and the key lies in utilizing the optimal state value $P_{\text {pbest }}$ experienced by the particles swarm and the state value $P_{\text {gbest }}$ of the maximum particle with the greatest fitness function value, and updating the speed and position of each particle on a real-time base through equation (10) and (11), accordingly forcing the particles to be closer to high likelihood area.

$$
\begin{aligned}
& V_{k}^{i}=\mid \text { Rand } n\left|\times\left(P_{\text {pbest }}-X_{k-1}^{i}\right)+\right| \text { rand } n \mid \times\left(P_{\text {gbest }}-X_{k-1}^{i}\right) \\
& X_{k}^{i}=X_{k-1}^{i}+V_{k-1}^{i}
\end{aligned}
$$

Where $\mid$ Rand $n \mid$ and $\mid$ rand $n \mid$ are positive Gaussian distribution random numbers.

\section{Building of Integrated Navigation Model}

\subsection{State and Measurement Equations}

The application of filter to GPS/INS integrated navigation system is ultimately intended for a more parameter ${ }^{[10]}$, and the selection of particle filter state normally resorts to indirect process, i.e., the error $\Delta \mathrm{X}$ of the navigation parameter outputted by a certain GPS/INS integrated navigation system is taken as PF's estimated state. While indirect process is used for state estimation process, the state of particle filter can be the combination of various errors in GPS/INS integrated navigation system, without participating in the computing processes of the GPS/INS integrated navigation parameters in navigation, such as the computing processes of INS' mechanical layout equation. Therefore, the state estimation process of particle filter is independent of the computation of the navigation parameters in original navigation system, and the INS will still have its strength of high update frequency fully revealed ${ }^{[11]}$.

Supposing the combination mode of GPS/INS integrated navigation system relies on the combination between target's attitude and velocity, measurement values can be divided into two values, namely, difference value of position and that of velocity. Difference value of position means that the difference between the longitude, latitude and altitude rendered by INS and the information of relevant position calculated by GPS receiver is figured out as measurement data. Difference value of velocity means that the difference between the information rendered by INS and the information of relevant velocity offered by GPS receiver is worked out as another style of measurement data.

The error state equation of integrated navigation system is shown as equation (12):

$$
\boldsymbol{X}(t)=\boldsymbol{F}(t) \boldsymbol{X}(t)+\boldsymbol{G}(t) \boldsymbol{W}(t)
$$

Where,

$$
X(t)=\left[\varphi_{E} \varphi_{N} \varphi_{U} \delta v_{E} \delta v_{N} \delta v_{U} \delta_{L} \delta_{\lambda} \delta_{h} \varepsilon_{b x} \varepsilon_{b y} \varepsilon_{b z} \varepsilon_{r x} \varepsilon_{r y} \varepsilon_{r z} \nabla_{x} \nabla_{y} \nabla_{z}\right]_{18 \times 1}^{T} \text {. }
$$


The position measurement data of INS can be defined as the sum of true value and corresponding error under the geographic coordinate system.

$$
\left[\begin{array}{l}
L_{I} \\
\lambda_{I} \\
h_{I}
\end{array}\right]=\left[\begin{array}{c}
L_{t}+\delta L \\
\lambda_{t}+\delta \lambda \\
h_{t}+\delta h
\end{array}\right]
$$

The position measurement of GPS receiver can be defined as the difference between true value and corresponding error under the geographic coordinate system.

$$
\left[\begin{array}{c}
L_{G} \\
\lambda_{G} \\
h_{G}
\end{array}\right]=\left[\begin{array}{l}
L_{t}-\frac{N_{N}}{R_{M}} \\
\lambda_{t}-\frac{N_{E}}{R_{N \cos L}} \\
h_{t}-N_{h}
\end{array}\right]
$$

Where $\lambda_{t}, L_{t}$, and $h_{t}$ stand for actual location, and $N_{E}, N_{N}$, and $N_{U}$ for the errors of GPS receiver in the eastward, northward and skyward directions.

The position measurement vector is defined as equation (15):

$$
Z_{p}(t)=\left[\begin{array}{l}
\left(L_{I}-L_{G}\right) R_{M} \\
\left(\lambda_{I}-\lambda_{G}\right) R_{N} \cos L \\
h_{I}-h_{g}
\end{array}\right]=\left[\begin{array}{l}
R_{M} \delta L+N_{N} \\
R_{N} \delta \lambda \cos L+N_{E} \\
\delta h+N_{U}
\end{array}\right] \equiv \boldsymbol{H}_{p}(t) \boldsymbol{X}(t)+\boldsymbol{V}_{p}(t)
$$

Where, $\left.\boldsymbol{H}_{p}=\left[\begin{array}{llllll}0_{3 \times 6} & \vdots & \operatorname{diag}\left[R_{M}\right. & R_{N} \cos L & 1\end{array}\right] \quad \vdots \quad 0_{3 \times 9}\right]_{3 \times 18}$

$$
\boldsymbol{V}_{p}=\left[\begin{array}{lll}
N_{N} & N_{E} & N_{U}
\end{array}\right]^{T}
$$

The variances of measurement noise are $\sigma_{p N}^{2}, \sigma_{p E}^{2}$, and $\sigma_{p U}^{2}$.

$$
\left\{\begin{array}{l}
\sigma_{p N}=\sigma_{p} \cdot H D O P_{N} \\
\sigma_{p E}=\sigma_{p} \cdot H D O P_{E} \\
\sigma_{p U}=\sigma_{p} \cdot H D O P
\end{array}\right.
$$

Where, $\sigma_{p}$ is the pseudo-range measurement error of GPS receiver.

The velocity measurement of INS can be defined as the sum of true value and corresponding velocity error under the geographic coordinate system.

$$
\left[\begin{array}{l}
v_{I N} \\
v_{I E} \\
v_{I U}
\end{array}\right]=\left[\begin{array}{l}
v_{N}+\delta v_{N} \\
v_{E}+\delta v_{E} \\
v_{U}+\delta v_{U}
\end{array}\right]
$$

Where $v_{E}, v_{N}$, and $v_{U}$ represent the true velocities along eastward, northward and skyward axes in the geographic coordinate system.

The velocity measurement information of GPS can be also defined as the difference between true value and corresponding velocity measurement error in geographic coordinate system

$$
\left[\begin{array}{l}
v_{G N} \\
v_{G E} \\
v_{G U}
\end{array}\right]=\left[\begin{array}{l}
v_{N}-M_{N} \\
v_{E}-M_{E} \\
v_{v}-M_{U}
\end{array}\right]
$$


Where $M_{N}, M_{E}$, and $M_{U}$ constitute the components of velocity errors of GPS receiver along three axes, namely, northward, eastward and skyward axes.

Below is the definition of velocity vector:

$$
Z_{p}(t)=\left[\begin{array}{c}
v_{I N}-v_{G N} \\
v_{I E}-v_{G E} \\
v_{I U}-v_{G U}
\end{array}\right]=\left[\begin{array}{l}
\delta v_{N}+M_{N} \\
\delta v_{E}+M_{E} \\
\delta v_{U}+M_{U}
\end{array}\right] \equiv \boldsymbol{H}_{v}(t) \boldsymbol{X}(t)+\boldsymbol{V}_{v}(t)
$$

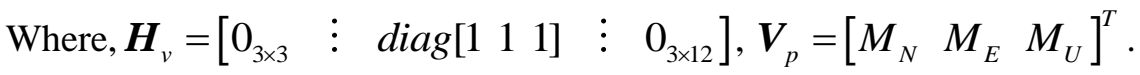

Assuming the measurement velocity of pseudo-range rate $\dot{\rho}$ of GPS receiver is $\sigma_{\dot{\rho}}^{2}$, the standard deviations of the eastward, northward and skyward velocity errors resulting from pseudo-range rate is as equation(20):

$$
\left\{\begin{array}{l}
\sigma_{v E}=H D O P_{E} \cdot \sigma_{\dot{\rho}} \\
\sigma_{v N}=H D O P_{N} \cdot \sigma_{\dot{\rho}} \\
\sigma_{v U}=V D O P \cdot \sigma_{\dot{\rho}}
\end{array}\right.
$$

The combination of position vector with velocity velocity can gain the measurement equation of position and speed integration system as equation(21):

$$
\boldsymbol{Z}(t)=\left[\begin{array}{l}
\boldsymbol{Z}_{p}(t) \\
\boldsymbol{Z}_{v}(t)
\end{array}\right]=\left[\begin{array}{l}
\boldsymbol{H}_{p} \\
\boldsymbol{H}_{v}
\end{array}\right] \boldsymbol{X}(t)+\left[\begin{array}{c}
\boldsymbol{V}_{p}(t) \\
\boldsymbol{V}_{v}(t)
\end{array}\right]=\boldsymbol{H}(t) \boldsymbol{X}(t)+\boldsymbol{V}(t)
$$

\subsection{Discretization of State and Measurement Equations}

Following result can be gained through the discretization of state equation (12) and measurement equation (21):

$$
\begin{gathered}
\boldsymbol{X}_{k}=\boldsymbol{\Phi}_{k, k-1} \boldsymbol{X}_{k-1}+\boldsymbol{\Gamma}_{k-1} \boldsymbol{W}_{k-1} \\
\boldsymbol{Z}_{k}=\boldsymbol{H}_{k} \boldsymbol{X}_{k}+\boldsymbol{V}_{k}
\end{gathered}
$$

Where, $\boldsymbol{\Phi}_{k, k-1}=\sum_{n=0}^{\infty}\left[F\left(t_{n}\right) T\right]^{n} / n !, \Gamma_{k-1}=\left\{\sum_{n=1}^{\infty} \frac{1}{n !}\left[F\left(t_{k}\right) T\right]^{n-1}\right\} G\left(t_{k}\right) T$.

As required by particle filter, the integrated navigation system and noises of state and measurement equations should be equipped with following characteristics:

$$
\begin{gathered}
E\left\{W(t) W^{\mathrm{T}}(\tau)\right\}=Q(t) \delta(t-\tau) \\
E\left\{V(t) V^{\mathrm{T}}(\tau)\right\}=R(t) \delta(t-\tau) \\
E\left\{W_{k} W_{j}^{\mathrm{T}}\right\}=Q_{k} \delta_{k j} \\
E\left\{V_{k} V_{j}^{\mathrm{T}}\right\}=R_{k} \delta_{k j}
\end{gathered}
$$

Where, $\delta_{k j}=\left\{\begin{array}{ll}1 & k=j \\ 0 & k \neq j\end{array},\left\{\begin{array}{l}Q_{k}=Q(t) / T \\ R_{k}=R(t) / T\end{array}\right.\right.$.

\section{DPSO-PF Algorithm}

\subsection{Improvement of DPSO-PF}

Based on the enlightenments brought by biological immune system and clone mechanism, an orthogonal immune clone partial swarm optimized algorithm is presented 
for optimizing function's solution. Firstly, let $A(k)=\left\{a_{1}(k), a_{2}(k), \cdots, a_{\text {size }}(k)\right\}$ denotes the $k$ the generation particle population, $S_{\text {size }}$ denotes the scale of particle swarm. Each particle can actualize particle swarm update through clone selection and self-learning. Corresponding operators are as follows:

(1) Immune clone operator. For any antibody $a_{i}=\left(a_{i, 1}, a_{i, 2}, \cdots, a_{i, n}\right)$ in particles $A(k)$ of $k$ th generation, $i=1,2, \cdots, S_{\text {size }}$ performs clone operation process with scale $q_{i}$, becoming clone $q_{i}$ of antibody $a_{i}$. The $q_{i}$ clone of antibody $a_{i}$ is defined as $\bar{a}^{i}=I_{i} \times a_{i}$, wherein $\bar{a}^{i}$ is the antibody group of $\bar{a}^{i}$ after cloning, and $I_{i}$ is the $q_{i}$ dimensional vector.

$$
q_{i}(k)=\operatorname{Int}\left[n_{c} \times \frac{f\left(a_{i}\right)}{\sum_{j=1}^{S_{\text {size }}} f\left(a_{j}\right)} \Upsilon\right], i=1,2, \cdots, S_{\text {size }}
$$

where $n_{c}$ denotes population clone scale, $n_{c}>S_{\text {size }}$, $\operatorname{Int}()$ means rounding up. $f\left(a_{i}\right)$ is the affinity of antibody $a_{i}$, while $\Upsilon$ reflects the affinity of antibody $a_{i}$ with other particles and is defined as equation(29):

$$
\Upsilon=\min \left\{D_{i j}\right\}=\min \left\{\exp \left(\left\|a_{i}-a_{j}\right\|\right)\right\},
$$

In which $\|\cdot\|$ denotes any of the norms, and in this paper Euclidean distance is employed. $D$ is a symmetric matrix reflecting the diversity of population.

(2) Immune gene operation. Immune gene operation consists of genetic recombination and genetic mutation mainly. To facilitate the communication of useful data among individuals and to enhance the uniformity of progeny particle, orthogonal recombination method is used to recombine the cloned particles by probability.

(3) Genetic recombination. Given the genetic recombination operator $P^{c}$, and $C(k)=\left\{c^{1}(k), c^{2}(k), \cdots, c^{S_{\text {SIIE }}}(k)\right\}$ as the particle swrm after recombination.

Taking two parental particles $b_{1}=\left(b_{1,1}, b_{1,2}, \cdots, b_{1, n}\right)$ and $b_{2}=\left(b_{2,1}, b_{2,2}, \cdots, b_{2, n}\right)$ into consideration, a sub-space $[l, u]$ is determined in accordance with the equation (30):

$$
\left\{\begin{array}{l}
l=\left[\min \left(b_{1,1}, b_{2,1}\right), \min \left(b_{1,2}, b_{2,2}\right), \cdots, \min \left(b_{1, n}, b_{2, n}\right)\right] \\
u=\left[\max \left(b_{1,1}, b_{2,1}\right), \max \left(b_{1,2}, b_{2,2}\right), \cdots, \max \left(b_{1, n}, b_{2, n}\right)\right]
\end{array}\right.
$$

Each of the area in Space $[l, u]$ is quantified into $Q$ levels, and the difference between two neighboring levels are always the same. $\beta_{i}=\left(\beta_{i, 1}, \beta_{i, 2}, \cdots, \beta_{i, Q}\right)$ is defined as equation (31):

$$
\begin{cases}\min \left(l_{i}, u_{i}\right), & j=1 \\ \min \left(l_{i}, u_{i}\right)+(j-1) \times\left(\frac{\left|l_{i}-u_{i}\right|}{Q-1}\right), & 2 \leq j \leq Q-1 \\ \max \left(l_{i}, u_{i}\right), & j=Q\end{cases}
$$

$F-1$ integer will be generated, $k_{1}, k_{2}, \cdots, k_{F-1}$, and $1<k_{1}<k_{2}<\cdots<k_{F-1}<n$ is met. Then for any particles $x=\left(x_{1}, x_{2}, \cdots, x_{n}\right)$, the following $F$ factors will be generated.

$$
\begin{aligned}
& f_{1}=\left(x_{1}, \cdots, x_{k 1}\right), f_{2}=\left(x_{k 1+1}, \cdots, x_{k 2}\right), \cdots, \\
& f_{F}=\left(x_{F-1+1}, \cdots, x_{n}\right)
\end{aligned}
$$


$Q$ levels of factor $f_{i}$ are

$$
\left\{\begin{array}{l}
f_{i}(1)=\left(\beta_{k_{i-1}+1,1}, \beta_{k_{i-1}+2,1}, \cdots, \beta_{k_{i}, 1}\right) \\
f_{i}(2)=\left(\beta_{k_{i-1}+1,2}, \beta_{k_{i-1}+2,2}, \cdots, \beta_{k_{i}, 2}\right) \\
\cdots \\
f_{i}(Q)=\left(\beta_{k_{i-1}+1, Q}, \beta_{k_{i-1}+2, Q}, \cdots, \beta_{k_{i}, Q}\right)
\end{array}\right.
$$

The orthogonal matrix $L_{M}\left(Q^{F}\right)=\left[r_{i, j}\right] M_{2} \times F$ is used to generate the $M$ particles:

$$
\left\{\begin{array}{l}
\left(f_{1}\left(r_{1,1}\right), f_{2}\left(r_{1,2}\right), \cdots, f_{F}\left(r_{1, F}\right),\right) \\
\left(f_{1}\left(r_{2,1}\right), f_{2}\left(r_{2,2}\right), \cdots, f_{F}\left(r_{2, F}\right),\right) \\
\cdots \\
\left(f_{1}\left(r_{M, 1}\right), f_{2}\left(r_{M, 2}\right), \cdots, f_{F}\left(r_{M, F}\right),\right)
\end{array}\right.
$$

Finally, an optimal particle is selected from $M$ particles as the clone progeny subject. Given $Q=3, F=4, M=9$, and orthogonal matrix is marked as $L_{9}\left(3^{4}\right)$.

(4) Genetic mutation. A E mutation strategy is used, letting $D(k)=\left\{d^{1}(k), d^{2}(k), \cdots, d^{S_{\text {SIZE }}}(k)\right\}$ the population after mutation:

$$
d_{j}^{i}=c_{j}^{i}-\frac{f i x\left(c_{j}^{i} \times 10^{l}(\bmod 10-m d(10))\right.}{10^{l}}
$$

$i=1,2, \cdots, S_{\text {SIZE }} ; j=1,2, \cdots, q$,

where $t$ is the random number of $0 \sim 15$, fix () is rounded down.

(5) Clone selection operator. Given $\forall i=1,2, \cdots, S_{\text {SIZE }}$, then:

$$
d_{i}^{\prime}(k)=\left\{d_{j}^{i}(k) \mid \min f\left(d_{j}^{i}\right), j=1,2, \cdots, q\right\}
$$

For probability $p^{k}\left(d_{i}^{\prime}(k) \bigcup a_{i}(k) \rightarrow a_{i}(k+1)\right)$,

$$
p^{k}\left(a_{i}(k+1)=d_{i}^{\prime}(k)\right)= \begin{cases}1, & f\left(a_{i}(k)\right)>f\left(d_{i}^{\prime}(k)\right) \\ \exp \left(-\frac{f\left(a_{i}(k)\right)-f\left(d_{i}^{\prime}(k)\right)}{a}\right), & f\left(a_{i}(k)\right) \leq f\left(d_{i}^{\prime}(k)\right) \text { and } \\ 0, & a_{i}(k) \text { is not the best antibody. } \\ & f\left(a_{i}(k)\right) \leq f\left(d_{i}^{\prime}(k)\right) \text { and } \\ & a_{i}(k) \text { is the best antibody. }\end{cases}
$$

(6) Self-learning operator. For the optimal individual $\hat{a}(k)=\left(\hat{a}_{1}(k), \hat{a}_{2}(k), \cdots, \hat{a}_{n}(k)\right)$, first of all a self-learning population $L$ is generated with size $L_{\text {SIZE }}$, then $L$ is as equation(38):

$$
L=\left\{\begin{array}{l}
\hat{a}(k), \quad i=1 \\
\text { New }_{i}, \text { others }
\end{array}\right.
$$

wherein $N e w_{i}=\left(e_{i, 1}, e_{i, 2}, \cdots, e_{i, n}\right)$ is generated according to the equation(39):

$e_{i, k}=\hat{a}_{i, k} \cdot U(1-$ sradius, $1+$ stradius $)$

$k=1,2, \cdots, n$, in which sradius $\in[0,1]$ denotes searching semi-diameter, $U(1-$ sradius, $1+$ sradius $)$ denotes the random number. Given $\operatorname{Min}_{i}=\left(m_{1}, m_{2}, \cdots, m_{n}\right)$ 
the individual with optimal fitness in $L$, e.g. $\operatorname{Min}_{i} \in L$, and for any individual $L_{i} \in L$ in a small population, we have $f\left(L_{i}\right) \geq f\left(\operatorname{Min}_{i}\right)$, if $L_{i}$ meets $f\left(L_{i}\right) \leq f\left(\operatorname{Min}_{i}\right)$, then it is a winner and can be preserved in the self-learning swarm, or otherwise a loser that should die. The eliminated individuals will be occupied by new particles New $w_{i}^{\prime}=\left(e_{i, 1}^{\prime}, e_{i, 2}{ }^{\prime}, \cdots, e_{i, n}{ }^{\prime},\right)$ generated by $\operatorname{Min}_{i}$.

$$
e_{i, k}^{\prime}= \begin{cases}m_{k}+d \times\left(m_{k}-L_{i, k}\right), k=1,2, \cdots, n . & \text { if } U(0,1)<P_{0} \\ \left(m_{1}, m_{2}, \cdots, m_{i_{1}-1}, m_{i_{2}}, m_{i_{2}-1}, \cdots,\right. & \text { else } \\ \left.m_{i_{1}+1}, m_{i_{1}}, m_{i_{2}+2}, \cdots, m_{n}\right) & \end{cases}
$$

where $d$ is the random variable within range $[0,1]$.

\subsection{Steps for DPSO-PF}

(1) Take $N$ particles $\left\{x_{0: k}^{i}, i=1, \ldots, N\right\}$ as samples from importance function at the initial time. The importance density function is defined as equation (41) :

$$
x_{k}^{i} \sim q\left(x_{k}^{i} \mid x_{k-1}^{i}, z_{k}\right)=p\left(x_{k}^{i} \mid x_{k-1}^{i}\right)
$$

Giving the fitness function is as equation (42):

$$
Y=\exp \left[-\frac{1}{2 R_{k}}\left(z_{\text {New }}-z_{\text {Pred }}\right)\right]
$$

Where $z_{\text {New }}$ is the latest observed value, $z_{\mathrm{Pr} e d}$ is the predictive observed value.

(2) Calculate the importance value:

$$
w_{k}^{i}=w_{k-1}^{i} p\left(z_{k} \mid x_{k-1}^{i}\right)=w_{k-1}^{i} p\left(z_{k} \mid x_{k}^{i}\right)
$$

(3) Updating the speed of particle swarm $A(k)$, then we have $A^{\prime}(k)$.

(4) Replace $T \%$ relatively undesirable individuals with $T \%$ desirable ones in $A^{\prime}(k)$, and $A(k) \leftarrow A^{\prime}(k)$.

(5) Perform clone operation against particle population $A(k)$, including clone recombination and clone mutation, then $\bar{A}(k)$.

(6) Perform immune gene operation against particle population $\bar{A}(k)$, then $D$.

(7) Perform clone selection against $D$, then $A^{*}(k)$.

(8) Force the self-learning operator to take effect on the optimal particle of $A^{*}(k)$ and then to upgrade the optimal particle.

(9) Setting $x_{e}$ is the individual extreme value of the current particle, $p b_{t}^{i}$ the optimal solution at $t$ time of particle ${ }^{i}$. gbest $_{k}$ is the global optimal solution at $t$ time .Compare their fitness, update $p b$ and $p g$ as equation (44) and (45):

$$
\begin{aligned}
& p b_{k}^{i}= \begin{cases}p b_{k}^{i}, & Y\left(x_{g}\right)<Y\left(p b_{k}^{i}\right) \\
x_{g}, & Y\left(x_{g}\right)>Y\left(p b_{k}^{i}\right)\end{cases} \\
& p g_{k} \in\left\{x_{k}^{1}, x_{k}^{2}, x_{k}^{3}, \cdots x_{k}^{N} \mid Y(x)\right\}=\max \left\{Y\left(x_{k}^{1}\right), Y\left(x_{k}^{2}\right), Y\left(x_{k}^{3}\right), \cdots Y\left(x_{k}^{N}\right)\right\}
\end{aligned}
$$

(10) When the optimal value complies with the initially-set threshold value $\varepsilon$ or DPSO-PF reached maximum iteration times $\lambda$, it is indicated that the particles have been already distributed around the true state. By now optimization will be stopped. Else jump to step (3).

(11) Calculate the weight of the particles after optimization and perform normalization: 


$$
\begin{aligned}
w_{k}^{i} & =w_{k-1}^{i} \frac{p\left(y_{k} \mid x_{k}^{i}\right) p\left(x_{k}^{i} \mid x_{k-1}^{i}\right)}{q\left(x_{k}^{i} \mid x_{k-1}^{i}, y_{k}\right)} \\
w_{k}^{i} & =w_{k}^{i} / \sum_{i=1}^{N} w_{k}^{i}
\end{aligned}
$$

(12) State output:

$$
x=\sum_{i=1}^{N} w_{k}^{i} x_{k}^{i}
$$

\section{Experimental Simulation}

\subsection{Simulation Test of Basic Algorithm Performance}

Using a univariate nonstationary growth model, the process equation and measurement equation of are given as equation (49) and (50):

$$
\begin{aligned}
& x(t)=0.5 x(t-1)+\frac{25 x(t-1)}{1+[x(t-1)]^{2}}+8 \cos [1.2(t-1)]+w(t) \\
& z(t)=\frac{x(t)^{2}}{20}+v(t)
\end{aligned}
$$

In which, $w(t)$ and $v(t)$ are zero-mean Gaussian noise. Since this model is highly non-linear and the likelihood function presents bimodal ${ }^{[20]}$.

The particle upgrade process of PSO-PF turns out to be an iterative optimization process. The concept of the optimization success rate $\eta$ is defined, with the calculation marking variable designed as temp, let the sampling time be rSTEP; if the iteration number reach the preset largest iteration number at the end of the time, then temp $=$ temp +1 ; however, the failure to reach the largest preset iteration number mean that the optimization is successful and the optimization success rate is defined as equation (51):

$$
\eta=\frac{r S T E P-t e m p}{r S T E P}
$$

By using PF、PSO-PF、DPSO-PF, tracking of this non-linear model are performed, and the formula of RMSE is

$$
R M S E=\left[\frac{1}{T} \sum_{t=1}^{T}\left(x_{t}-x_{t}\right)^{2}\right]^{1 / 2}
$$

Giving the particle number $N=100$, and system noise variance $Q=10$, measurement noise variance $R=1$, the simulation result is showed in Figure 1 and Figure 2. After 300 times of Monte-Carlo simulation, the data is given in Table 1 . 


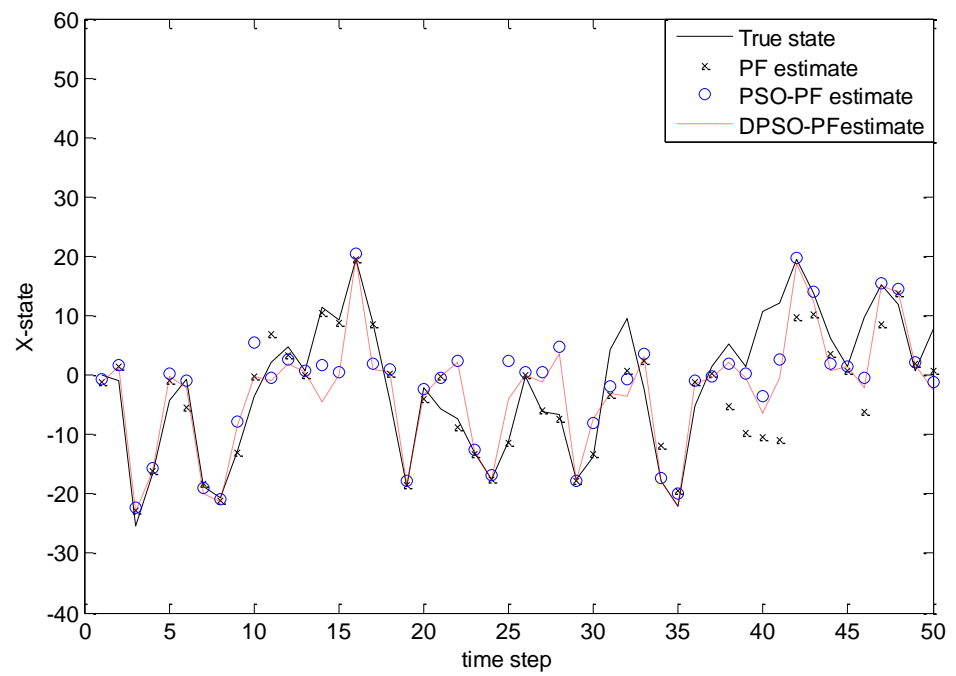

Figure 1. State Estimation of Different Algorithm（ $Q=10 ）$

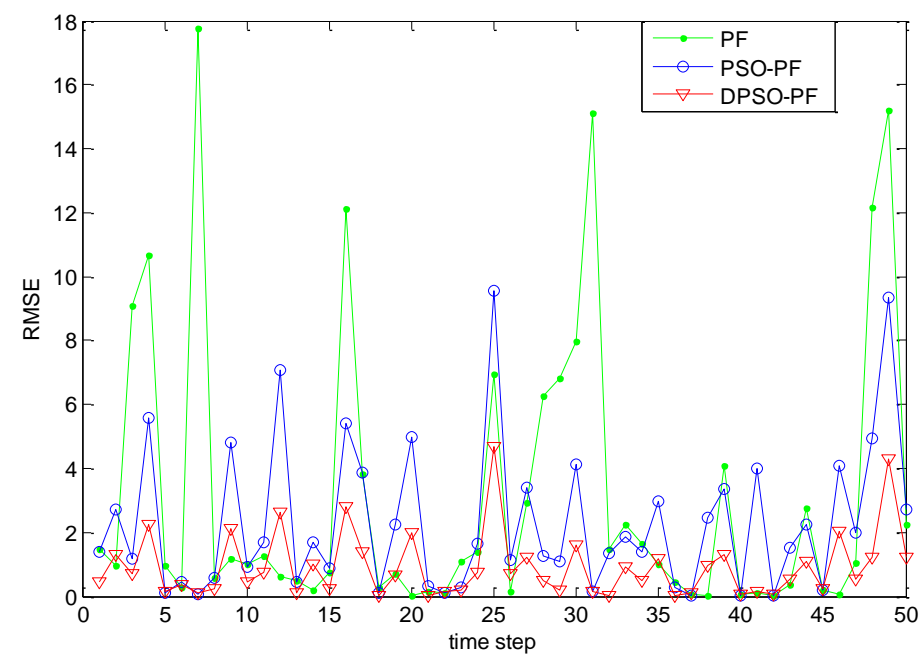

Figure 2. RMSE of Different Algorithm $(Q=10)$

(2) Giving the particle number $N=100$, and $R=1, Q=20$, the simulation result is showedin Figure 3 and Figure 4. After 300 times of Monte-Carlo simulation, the data is given in Table 1. 


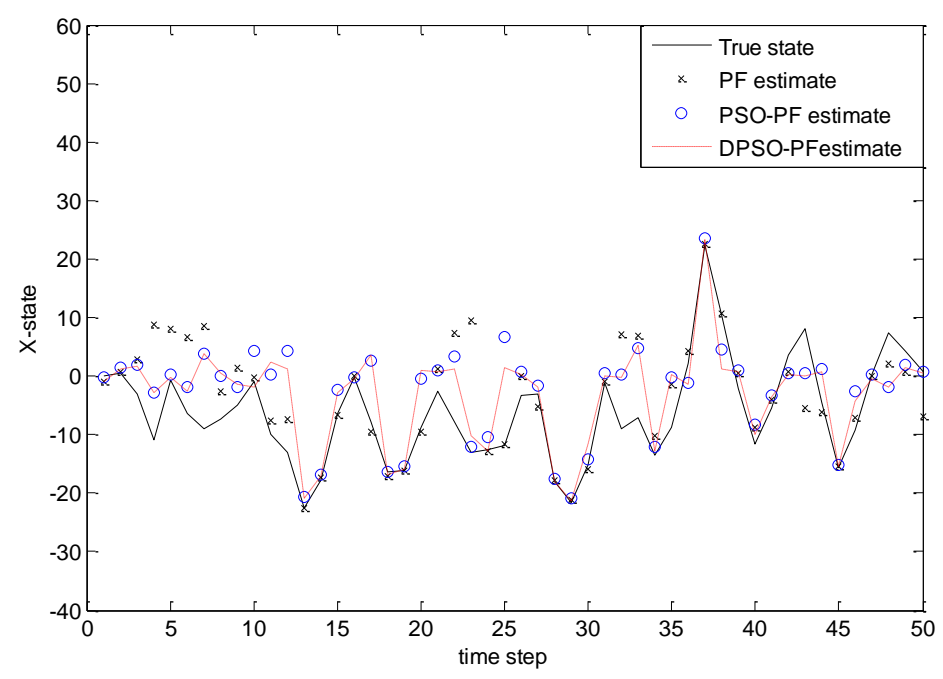

Figure 3. State Estimation of Different Algorithm $(Q=20)$

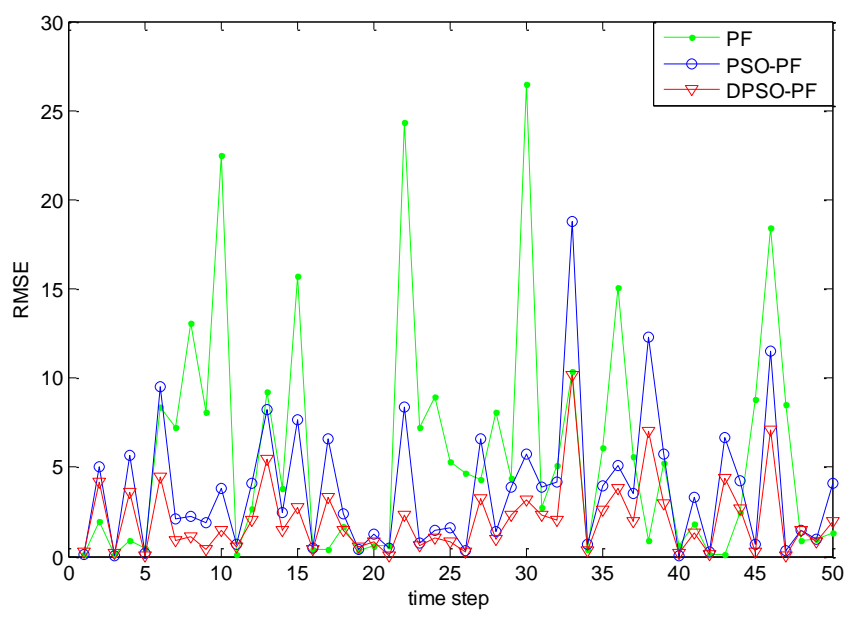

Figure 4. RMSE of Different Algorithm $(Q=20)$

Table 1. Comparison of Simulation Parameters by UNGM Model

\begin{tabular}{ccccc}
\hline Parameters & Algorithms & $\eta / \%$ & RMSE & Time/s \\
\hline$Q=1, N=200$ & PF & $/$ & 3.5892 & 0.6684 \\
$Q=1, N=50$ & PSO-PF & 97.83 & 2.4563 & 0.5821 \\
$Q=1, N=50$ & DPSO-PF & 99.17 & 1.4521 & 0.5259 \\
$Q=20, N=200$ & PF & $/$ & 5.9635 & 0.6854 \\
$Q=20, N=50$ & PSO-PF & 97.72 & 4.2155 & 0.5915 \\
$Q=20, N=50$ & DPSO-PF & 99.19 & 2.4287 & 0.5502
\end{tabular}

As shown by the Figure 1 to Figure 4 and table 1, the error of the integration with PSO$\mathrm{PF}$ is significantly lower than that of standard PF, and the integration is in fact the particle 
optimization of particle swarm optimization that can improve particles quality. This can be ascribed to the fact that the orthogonal cross strategy can reinforce the uniformity of progeny particles, avoiding loss of optimal solution in the individual neighborhood. At the same time the precocity of individuals can be eliminated, and thereby the speed and precision of the improved particle filter is enhanced. In the above calculation, with respect to both estimation precision and operation time, DPSO-PF has the most desirable performance.

\subsection{Simulation Test of Performance In Integrated Navigation System}

Let the latitude and longitude of the initial position of system state vector be $32^{\circ}$ and $118^{\circ}$, respectively; the random and constant drift errors of the gyroscope be $0.05^{\circ} / \mathrm{h}$,

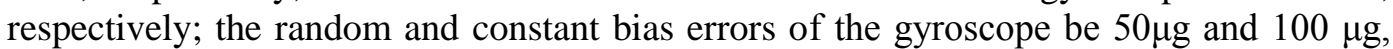
respectively; the update cycle of inertial navigation be $0.01 \mathrm{~s}$; the cycle of KF be $1 \mathrm{~s}$; and the experiment time be 500s. In the paper, an analysis is implemented on the position and velocity error curves along northward, eastward and skyward directions before and after the integrated particle filter correction.
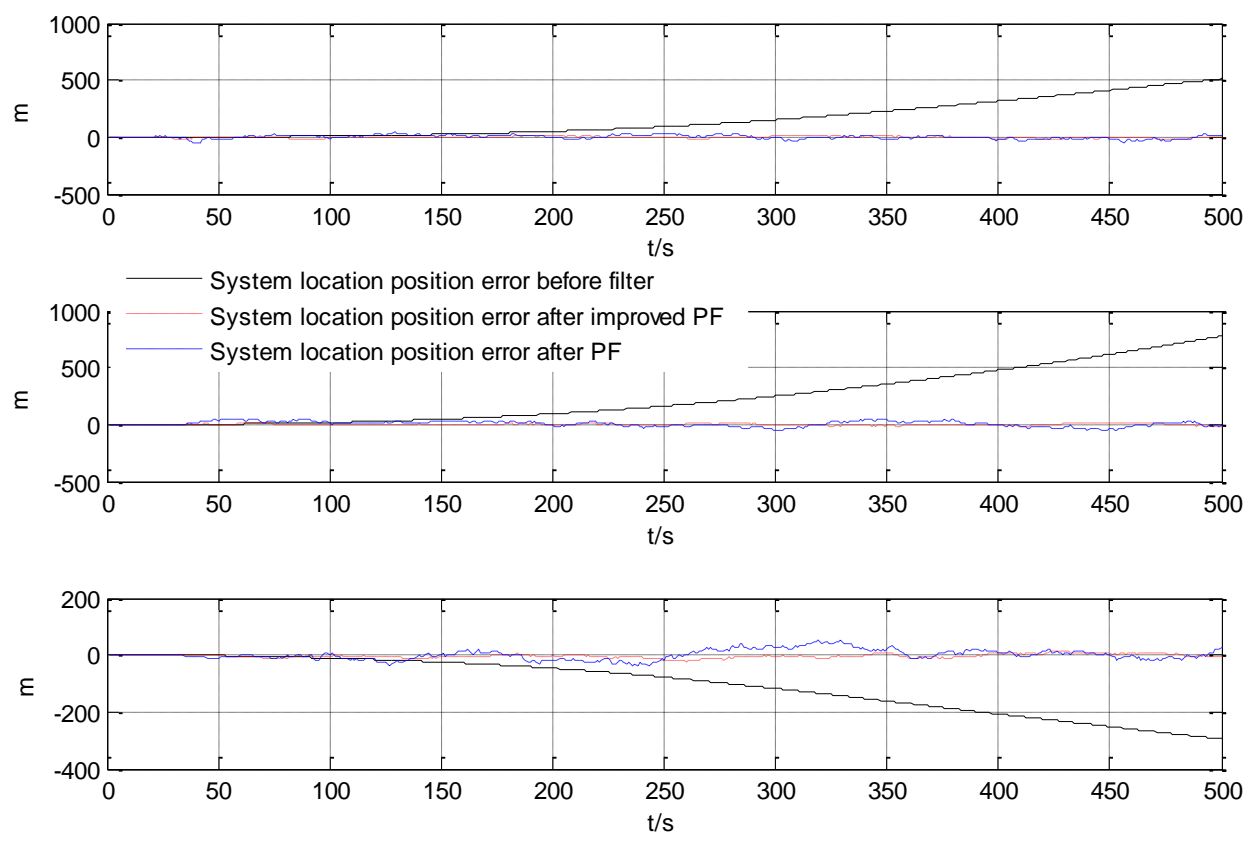

Figure 5. Position Error in Different Directions (Northward, Eastward, Skyward) 

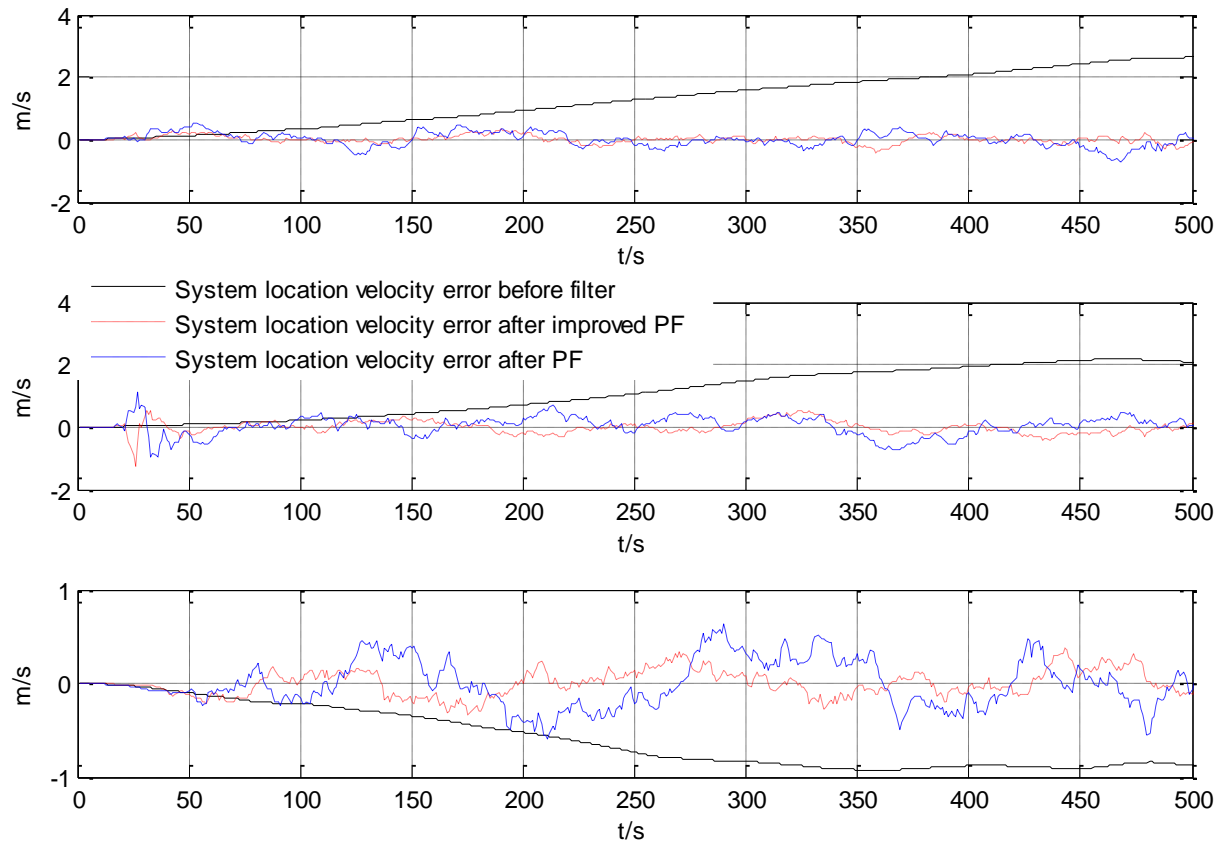

\section{Figure 6. Velocity Error in Different Directions (Northward, Eastward, Skyward)}

As illustrated by the Figure.5 and figure.6, GPS/INS integrated navigation system faces rapid divergence prior to the application of filter, but the parameter errors of the system are correctly effectively upon the use of DPSO-PF. According to the simulation result, integrated filter can control the position errors within $33.73 \mathrm{~m}$, with the mean square deviations of position errors along the northward, eastward and skyward directions being $6.80 \mathrm{~m}, 7.48 \mathrm{~m}$, and $5.23 \mathrm{~m}$, respectively, and those of velocity errors being $0.24 \mathrm{~m} / \mathrm{s}, 0.21$ $\mathrm{m} / \mathrm{s}$ and $0.19 \mathrm{~m} / \mathrm{s}$, respectively. As for DPSO-PF, since it successfully preserves the diversity of population, it has the higher precision and can well estimate the position and velocity.

\section{Conclusion}

This paper brings forward a novel particle filter algorithm based on based on dynamic clone particle swarm which can enhance the convergence speed and grantee the population diversity while enlarge the local searching range, thereby improving the quality of the particles' neighborhood, and then a good balance will be reached between search ability and convergence speed, The experimental results indicate that the improved particle filter improves the precision and thus of high applicable value in GPS/INS integrated navigation system.

\section{Acknowledgments}

This work is partially supported by the National Natural Science Foundation of China (61501521); National Natural Science Foundation of China (U1330133); National Natural Science Foundation of China (61473153); National Natural Science Foundation of China (61403421); National Natural Science Foundation of China (61203266); Key Defense Advanced Research Project of China (40405070102). Thanks for the help. 


\section{References}

[1] D. Bhatt, P. Aggarwal and V. Devabhaktuni, "A novel hybrid fusion algorithm to bridge the period of GPS outages using low-cost INS", Expert Systems with Applications, vol.41, no.5, (2014), pp.21662173.

[2] Ben K. H. Soon, Steve Scheding and Hyung-KuenLee, "An approach to aid INS using time-differenced GPS carrier phase (TDCP) measurements", GPS Solution, vol.12, no.4, (2008), pp.261-271.

[3] M. A. K. Jaradat and M. F. Abdel-Hafez. Enhanced, "Delay Dependent, Intelligent Fusion for INS/GPS Navigation System”, IEEE Sensors Journal, vol.14, no.5, (2014), pp.1545 - 1554.

[4] S.Y. Cho, "IM-filter for INS/GPS-integrated navigation system containing low-cost gyros", IEEE Transactions on Aerospace and Electronic Systems, vol.50, no.4, (2014), pp.2619 - 2629.

[5] X. Wang and C. Z. Han, "A multiple model particle filter for maneuvering target tracking based on composite sampling", Acta automatica sinica, vol. 39, no. 7, (2013), pp. 1152-1156.

[6] H. W. Li, J. Wang and H. T. Wang, "A New Particle Filter Based on Differential Evolution Method", Journal of Electronics \& Information Technology, (2011), pp. 1639-1643.

[7] F. S. Jian, Y. M. Xu and Z. J. Yin, "Enhanced multiple model particle filter for maneuvering target tracking", Control Theory \& Applications, (2010), pp. 1012-1016.

[8] K. Hwang and W. Sung, "Load balanced resampling for real-time particle filtering on graphics processing units", IEEE Transactions on Signal Process, vol. 61, no. 2, (2013), pp. 411-419.

[9] Z. Fang, G. F. Tong and X. H. Xu, "Particle swarm optimized particle filter" Control and Decision, vol.22, no.3, (2007), pp. 273-277.

[10] C. Hide, T. Moore and M. Smith, "Adaptive Kalman Filtering for Low-cost INS/GPS" Journal of Navigation, vol.56, no.1, (2003), pp.143-152.

[11] W. Ding, J. Wang and C. Rizos, "Improving Adaptive Kalman Estimation in GPS/INS Integration" The Journal of Navigation, vol.60, no.3, (2007), pp. 517-529.

[12] Z. M. Chen, Y. M. Bo, P. L. Wu and W. J. Zhou, "A new particle filter based on organizational adjustment particle swarm optimization”, Applied Mathematics \& Information Science, vol.7, no.1, (2013), pp.179-186.

[13] M. H. Zhang, M. Xin and J. Yang, "Adaptive multi-cue based particle swarm optimization guided particle filter tracking in infrared videos", Neurocomputing, vol. 122, (2013), pp. 163-171.

[14] J. Zhao and Z. Li, "Particle filter based on Particle Swarm Optimization resampling for vision tracking", Expert Systems with Applications, vol.37, no.12, (2010), pp.8910-8914.

[15] M. H. Zhang, M. Xin and J. Yang, "Adaptive multi-feature tracking in particle swarm optimization based particle filter framework", Journal of Systems Engineering and Electronics, vol. 23, no. 5, (2012), pp. 775-783.

[16] Z. M. Chen, Y. M. Bo, P. L. Wu and S. L. Yu, "A particle filter algorithm based on chaos particle swarm optimization and its application to radar target tracking", Journal of Computational Information Systems. vol.8, no.7, (2012), pp.3081-3090.

[17] W. Xian, B. Long, M. Li and H. Wang, "Prognostics of Lithium-Ion Batteries Based on the Verhulst Model, Particle Swarm Optimization and Particle Filter", IEEE Transactions on Instrumentation and Measurement, vol. 63, no. 1, (2014), pp. 2-17.

[18] Y. Li, B. Bai and Y. Zhang, "Improved particle swarm optimization algorithm for fuzzy multi-class SVM", Journal of Systems Engineering and Electronics, vol. 21, no. 3, (2010), pp. 509-513.

[19] S.A. MirHassani and N. Abolghasemi, "A particle swarm optimization algorithm for open vehicle routing problem”, Expert Systems with Applications, vol.38, no.9, (2011), pp.11547-11551.

[20] H.W. Li and J. Wang, "Particle filter for manoeuvring target tracking via passive radar measurements with glint noise", IET Radar, Sonar \& Navigation, vol. 6, no. 3, (2012), pp. 180-189. 


\section{Authors}
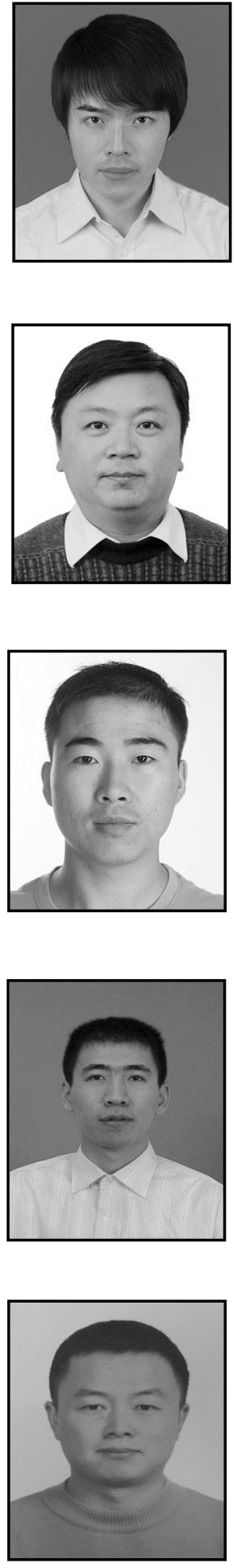

Zhimin Chen, he received B.S., M.S. and Ph.D degree in automation from Nanjing University of Science and Technology in 2008, 2010 and 2014. He is currently Post-doctor of China Satellite Maritime Tracking and Controlling Department. His research interests are in the areas of target tracking, control theory and control applications, intelligent optimization algorithm.

Yuming Bob, he received the B.S., M.S. and Ph.D degree in automation from Nanjing University of Science and Technology in 1984, 1990 and 1996. He is currently a professor, PhD supervisor in Nanjing University of Science and Technology and China Satellite Maritime Tracking and Controlling Department. His research interests are in the areas of Control theory and control applications.

Yongliang Zhang, he received the B.S., M.S. and Ph.D. degrees in information and communication engineering from national university of defense and technologys in 2000, 2004 and 2010. He is currently an assistant professor of China Satellite Maritime Tracking and Controlling Department. His research interests are in the areas of control theory and target tracking.

Yujian Li, he received the B.S., M.S. and Ph.D. degrees in theoretical physics from University of Science and Technology of China in 2006, 2008 and 2011. He is currently an assistant professor of China Satellite Maritime Tracking and Controlling Department. His research interests are in the areas of control theory and target tracking.

Xiaodong Ling, he received the B.S., M.S. and Ph.D. degrees in automation from national university of defense and technology in 2000, 2010 and 2014. He is currently an associate professor of China Satellite Maritime Tracking and Controlling Department. His research interests are in the areas of intelligent optimization algorithm and target tracking. 
International Journal of Control and Automation Vol. 9, No. 10 (2016) 